\title{
ON THE CONTINUITY AND LIMITING VALUES OF FUNCTIONS
}

\author{
BY \\ ISTVÅN S. GÁL
}

1. Introduction and background. In the present paper we shall deal with functions $f$ which map an abstract set $X$ into a uniform space $Y$. We assume that certain topological structures are given on the set $X$ so that we can speak of the limting values of $f$ as the independent variable $\xi$ approaches $x$ and we can also define the continuity of $f$ at some of the points $x \in X$.

These topological structures on $X$ will be such that for a noncountable set of points $x \in X$ the limiting values of $f$ as $\xi \rightarrow x$ and the continuity of $f$ at $x$ can be interpreted in at least two different ways. For example if $X$ is the real line one an speak about the limiting values of $f$ as $\xi$ approaches $x$ from the left and from the right, and of $\lim _{\xi \rightarrow x-0} f(\xi)$ and $\lim _{\xi \rightarrow x+0} f(\xi)$, and of the left and right continuity of $f$ at $x$. The exact definition of these topological structures on $X$ is given later.

We shall prove two general theorems for such functions: One of these concern the equality of the $\operatorname{limits} \lim f(\xi)$ as $\xi$ approaches the same point $\xi$ under different conditions. Two cases can be distinguished according as $\lim f(\xi)$ is uniquely determined for each of these topological structures or not. The other theorem states that except for a negligible subset of $X$ the function $f$ is continuous with respect to both or neither of the topologies defined on $X$.

These theorems include many old and new results as special cases from the theory of functions of one and of several real variables. For better understanding it is perhaps more suitable to discuss first some of the known results and to state the new ones afterwards. For real valued functions of one real variable some precise results of this type have been known for a long time. The common source of one group of these results is the following theorem due to W. H. Young [1]:

The set of those points $x$ for which $\lim \sup _{\xi \rightarrow x-0} f(\xi) \neq \lim \sup _{\xi \rightarrow x+0} f(\xi)$ is countable.

The uses of this theorem can be illustrated by mentioning a few immediate consequences. Here it is convenient to use the expression "nearly everywhere" to abbreviate the phrase "with the possible exception of at most enumerably many points."

If $f$ is upper semicontinuous from the left or from the right nearly everywhere in an interval I then $f$ is upper semicontinuous nearly everywhere in $I$.

If $f$ is left or right upper semicontinuous and also left or right lower semi-

Presented to the Society December 27, 1956; received by the editors July 30, 1956. 
continuous nearly everywhere in I then $f$ is continuous nearly everywhere in $I$.

The direction of approach of the one sided semicontinuity may vary from point to point. An analogue of the first result can be obtained by interchanging the words upper by lower. The second corollary implies immediately the result that monotonic functions are continuous nearly everywhere.

Another less immediate consequence of Young's theorem is the result on the continuity of regular functions $[2 ; 3]$. We may as well state the result for a slightly wider class of functions: We say that $f$ is nearly regular in $I$ if the one sided $\operatorname{limits}_{\lim _{\xi \rightarrow x-0}} f(\xi)$ and $\lim _{\xi \rightarrow x+0} f(\xi)$ exist nearly everywhere in $I$. A great number of results on regular functions hold also for nearly regular functions. Using Young's theorem and a lemma which appears in the same paper by Young we obtain: in $I$.

If $f$ is nearly regular in the interval I then $f$ is continuous nearly everywhere

The auxiliary theorem concerns the interlimits and intercontinuity of $f$ : A real number $y$ is called an interlimit of $f$ as $\xi \rightarrow x$ if given any $\epsilon>0$ and any neighborhood $N_{x}$ of $x$ there is a $\xi \in N_{x}$ such that $|f(\xi)-y|<\epsilon$. The function $f$ is called intercontinuous at $x$ if $f(x)$ is an interlimit of $f$ at $x$. Young's auxiliary theorem states:

Every real valued function $f$ defined on an interval I is intercontinuous nearly everywhere in $I$.

A recent generalization of this result to functions $f$ which map one finite dimensional Euclidean space into another one is due to Bonferroni [4]. We shall extend this auxiliary theorem to uniform structures satisfying the first axiom of countability and an additional hypothesis which will be stated later.

A second group of theorems on real valued functions of a real variable concerns the relative magnitude of the Dini derivatives and one sided derivatives of such functions. The best known among these results is the theorem which states that any such function can have at most enumerably many cusps, i.e. the set of those points where the left-hand and right-hand derivatives exist and are different is countable. More generally we have $D_{-} \phi \leqq D^{+} \phi$ and $D^{-} \phi \geqq D_{+} \phi$ nearly everywhere [5].

The foregoing results on the extreme derivatives of $\phi$ can be interpreted as properties of the function $f$ of two real variables $x$ and $\xi$ defined as $f(x, \xi)$ $=(\phi(\xi)-\phi(x)) /(\xi-x)$ for every point $(x, \xi)$ with $x \neq \xi$. Blumberg [6] was the first to realize that these properties are independent of the special nature of the function $f$ and they hold for arbitrary functions of two real variables. His result was improved and extended by Miss Schmeiser [7] and Jarnik [8]. One of our main results gives a generalization of these theorems to abstract topological spaces.

2. Preliminary definitions and lemmas. Let us assume that $X$ is a fixed noncountable point set whose elements we shall denote by $x, x_{0}$ and $\xi$. There are various ways to describe topological structures on $X$ such that the phrase 
" $\xi$ tends to $x$ " or the equivalent symbol " $\xi \rightarrow x$ " becomes meaningful for the points $\xi \in X$ and for some points $x \in X$. Perhaps it is more suitable for our purposes to use the concept of a filter to describe convergence on $X$.

If the reader has a preference for nets he may replace the words "filter" by the words "net" in the statements of the theorems and in the proofs; there are only a few points in the proofs where the reasoning must be slightly modified. For the definitions and the equivalence of such concepts he can consult the articles of McShane $[9 ; 10]$ and Bartle [11]. An extensive bibliography can be found in [12]. For the sake of completeness and also to avoid confusion we state the definition of a filter:

$A$ filter $\mathcal{F}$ in the set $X$ is a nonvoid family of subsets of $X$ such that

(1) if $F_{1} \in \mathcal{F}$ and $F_{1} \subseteq F_{2}$ then $F_{2} \in \mathcal{F}$,

(2) if $F_{1}, F_{2} \in \mathcal{F}$ then $F_{1} \cap F_{2} \in \mathcal{F}$,

(3) if $F \in \mathcal{F}$ then $F$ is not empty.

A collection $Q$ of nonvoid subsets of $X$ is called a filter base if $B_{1}, B_{2}, \in B$ implies the existence of a $B \in B$ such that $B \subseteq B_{1} \cap B_{2}$. The family $\mathcal{F}$ of all subsets $F$ of $X$ which contain at least one set $B$ of $B$ is a filter in $X$, it is called the filter generated by $B$. It is easy to see that a subset $B$ of a filter $\mathcal{F}$ is a base of $\mathcal{F}$ if and only if every $F \in \mathcal{F}$ contains some $B \in ß$. If a filter $\mathcal{F}$ has a countable base then we say that $\mathcal{F}$ satisfies the first axiom of countability.

A topology $\mathfrak{J}$ on a set $A$ will be called a perfect topology if every noncountable subset $A^{*}$ of $A$ has a point of accumulation with respect to $J$ which belongs to $A^{*}$. For instance every Hausdorff topology satisfying the second axiom of countability is a perfect topology. For assume that $A^{*}$ is such that no point $\alpha \in A^{*}$ is a point of accumulation of $A^{*}$. Then there is a neighborhood $N_{\alpha}$ about each $\alpha \in A^{*}$ such that $N_{\alpha}$ contains finitely many elements of $A^{*}$. Then $\left\{N_{\alpha}\right\}$ covers $A^{*}$ and so by Lindelöf's theorem it has a countable subcovering. Since each $N_{\alpha}$ contains only a finite number of $\alpha^{\prime}$ s from $A^{*}$ it follows that $A^{*}$ is a countable set.

We shall use the notions of a uniform structure $\mathcal{U}$ and a uniform space $Y$. If the reader is not interested in such generalities he may assume that the space $Y$ which occurs in Theorems $1-4$ is a metric space. The proofs of these theorems are simpler if $Y$ is a metric space; instead of choosing a suitable "vicinity" $V$ it is sufficient to select a suitable $\epsilon>0$ and instead of finding a $W$ such that $W \circ W \subseteq V$ it is sufficient to take $\epsilon / 2$. Although all uniform spaces which occur in the sequel are pseudo-metric spaces we insist on using a uniform structure instead of a pseudo-metric. The reason for this is that several of the results can be extended almost without change to a more general situation where countable sets are replaced by sets having cardinality at most $m$ and noncountable sets by sets whose cardinality is strictly greater than $m$. In this case $Y$ need not be a pseudo-metric space; it is sufficient to suppose that $Y$ is a uniform space which has a structure base whose cardinality is at most $m$. The only essential change is in the definition of the topology $\mathfrak{J}$. 
Namely we must assume that every subset $A^{*}$ whose cardinality card $A^{*}>m$ has an $m$-accumulation point which belongs to $A^{*}$.

The usual definition of a uniform structure is as follows. (See for example [13], [14] or [15].)

$A$ uniform structure $u$ on a set $Y$ is a filter in the product $Y \times Y$ such that

(4) if $V \in \mathcal{u}$ then $\Delta \subseteq V$,

(5) if $V \in \mathcal{U}$ then $V^{-1} \in \mathcal{U}$,

(6) if $V \in \mathcal{U}$ then there is a $W \in \mathcal{U}$ such that $W \circ W \subseteq V$.

Here $\Delta$ denotes the diagonal of $Y \times Y$, i.e. $\Delta$ is the set of pairs $(y, y)$. The set $V^{-1}$ is the set of all pairs $\left(y_{2}, y_{1}\right)$ with $\left(y_{1}, y_{2}\right) \in V$. Moreover $W \circ W$ is the set of those pairs $\left(y_{1}, y_{3}\right)$ for which there is a suitable $y_{2} \in Y$ with $\left(y_{1}, y_{2}\right) \in W$ and $\left(y_{2}, y_{3}\right) \in W$. Axioms (4), (5), (6) correspond to the axioms $d(y, y)=0$, $d\left(y_{1}, y_{2}\right)=d\left(y_{2}, y_{1}\right), d\left(y_{1}, y_{3}\right) \leqq d\left(y_{1}, y_{2}\right)+d\left(y_{2}, y_{3}\right)$ of a metric space $Y$. The sets $V$ of the filter are called vicinities $\left({ }^{1}\right)$; in a metric space the $\epsilon$-vicinity is the set of those pairs $\left(y_{1}, y_{2}\right)$ for which $d\left(y_{1}, y_{2}\right)<\epsilon$. The uniform structure $u$ is said to satisfy the first axiom of countability if the filter $\mathcal{U}$ has a countable base.

We shall use the following lemmas:

Lemma 1. Given any $V \in \mathcal{U}$ there is a symmetric vicinity $W$ such that $W \circ W \subseteq V$.

Proof. $W$ is called symmetric if $W^{-1}=W \cdot V \cap V^{-1}$ is symmetric and by (5) and (2) we have $V \cap V^{-1} \in \mathcal{U}$ for any $V \in \mathcal{U}$. By (6) there is a $U \in \mathcal{U}$ such that $U \circ U \subseteq V \cap V^{-1}$ and so by the symmetry of $V \cap V^{-1}$ we have $U^{-1} \circ U^{-1}$ $=(U \circ U)^{-1} \subseteq V^{-1} \cap V$. This implies that $U \subseteq U \circ U \subseteq V \cap V^{-1}$ and $U^{-1} \subseteq U^{-1} \circ U^{-1} \subseteq V \cap V^{-1}$ so that $W=U \cap U^{-1} \subseteq V \cap V^{-1}$. $W \in \mathcal{U}$ is symmetric and $W \circ W \subseteq U \circ U \subseteq V$ because $U \circ U \subseteq V \cap V^{-1} \subseteq V$. This completes the proof.

Lemma 2. If $u$ satisfies the first axiom of countability then there exists a decreasing sequence $\left\{U_{n}\right\}$ of symmetric vicinities which form a basis of $\mathcal{u}$.

Proof. By hypothesis $\mathcal{u}$ has a countable basis, say $\left\{V_{n}\right\}$. By (5), (2) and (1) we have $W_{n}=V_{n} \cap V_{n}^{-1} \in U$ for every $n$. The sets $U_{n}=W_{1} \cap \cdots \cap W_{n}$ are clearly symmetric and by (2) they belong to $U$. Since $U_{n} \subseteq W_{n} \subseteq V_{n}$ and $\left\{V_{n}\right\}$ is a basis of $u$ the decreasing sequence $U_{1} \supseteq \cdots \supseteq U_{n} \supseteq \cdots$ of symmetric vicinities is also a basis of $\mathcal{u}$.

Every uniform structure $u$ determines a topology on $Y$ which is called the uniform topology derived from $u$ : Given any $y \in Y$ we associate with every $V \in \mathcal{U}$ a neighborhood $N_{\nu}$ of $y$ defined as $N_{\nu}=[\eta /(y, \eta) \in V]$. If $u$ has a countable base then the uniform topology satisfies the first countability axiom.

LEMMA 3. If the uniform space $Y$ defined by the uniform structure $u$ contains a countable set which is everywhere dense in $Y$ then for every $V \in \mathcal{U}$ there

(1) The names surrounding and uniformity are also used. 
is a subdivision $\mathfrak{\Re}_{V}$ of $Y$ into countably many disjoint sets $M_{V}^{k}$, called the meshes of $\mathscr{N}_{V}$, which satisfy $M_{V}^{k} \times M_{V}^{k} \subseteq V$.

Note. If $Y$ is a metric space containing a countable set which is dense everywhere in $Y$ the existence of such subdivisions $\mathscr{X}$ is well known $[5, \mathrm{p} .153]$, $[16$, pp. 133-134]. The name subdivision is used here to avoid confusions in terminology.

Proof. Let $\left\{y_{n}\right\}$ be dense in $Y$. Using Lemma 1 we can choose a symmetric $W \in U$ such that $W \circ W \subseteq V$ and consider the neighborhood $N_{n}$ of $y_{n}$ defined as $N_{n}=\left[y /\left(y_{n}, y\right) \in W\right]$. Since $\left\{y_{n}\right\}$ is everywhere dense in $Y$ the neighborhood $N=[\eta /(y, \eta) \in W]$ of $y$ contains some $y_{n}$. The symmetry of $W$ implies that $y \in N_{n}$ and so the neighborhood system $\left\{N_{n}\right\}$ covers $Y$. Clearly the symmetry implies that $\left(y^{\prime}, y^{\prime \prime}\right) \in W \circ W$ for any $y^{\prime}, y^{\prime \prime} \in N_{n}$ and so $N_{n} \times N_{n} \subseteq V$. The sets $M_{V}^{1}=N_{1}, M_{V}^{2}=N_{2}-M_{V}^{1} \cap N_{2}, \cdots, M_{V}^{k}=N_{k}-\left(M_{V}^{1} \cup \cdots \cup M^{k-1}\right) \cap N_{k}$, . . a are disjoint, their union is $Y$ and since $M_{V}^{k} \subseteq N_{k}$ we have $M_{V}^{k} \times M_{V}^{k} \subseteq V$. This completes the proof.

Given a function $f$ which maps the set $X$ into the topological space $Y$ and given a filter $\mathcal{F}$ in $X$ we say that $y \in Y$ is a cluster point or interlimit of $f$ with respect to $\mathcal{F}$ if for every $N_{y} ; y=f(x)$ and for every $F \in \mathcal{F}$ there is a $\xi \in F$ such that $f(\xi) \in N_{y}$. The set of all cluster points of $f$ with respect to $\mathcal{F}$ will be denoted by $f[\mathfrak{F}]$. It is easy to see that $f[\mathfrak{F}]$ is a closed set in $Y$ for every filter $\mathcal{F}$ in $X$.

A point $y \in Y$ is called a limit point of $f$ with respect to $\mathcal{F}$ if for every $N_{y}$ there is an $F \in \mathcal{F}$ such that $f(\xi) \in N_{y}$ for every $\xi \in F$. We say that $f$ is continuous at $x$ with respect to $\mathcal{F}$ if for every $N_{y}$ there is an $F \in \mathcal{F}$ such that $f(\xi) \in N_{y}$ for every $\xi \in F$. If $Y$ is a Hausdorff space then there exists at most one limit point of $f$ with respect to $\mathscr{F}$; if this limit point $y=(\mathscr{F}) \lim f(\xi)$ exists then $f[F]$ consists of the single point $y$.

Often these concepts are defined only when $X$ is a topological space and $\mathcal{F}$ is a filter which has exactly one limit point. By a limit point of $\mathcal{F}$ we understand any point $x$ such that every neighborhood $N_{x}$ of $x$ contains at least one $F \in \mathcal{F}$. Clearly $x$ is a limit point of $\mathcal{F}$ if and only if $\mathcal{P}_{x} \subseteq \mathcal{F}$ where $\mathcal{P}_{x}$ denotes the filter generated by the set of all open sets containing $x$. If $f(x)$ is an interlimit of $f$ with respect to the filter $\mathcal{P}_{x}$ then $f$ is called intercontinuous at the point $x$. The limit superior of a real valued $f$ with respect to a filter $\mathcal{F}$ is defined as

$(\mathfrak{F}) \lim \sup f(\xi)=(\mathscr{F}) \lim [(F) \operatorname{lub} f(\xi)]$

where $(F)$ lub $f(\xi)$ denotes the supremum of $f(\xi)$ on the set $F$.

3. Main results. Now we are ready to formulate our main theorems. For throughout this section and also in the forthcoming proofs let $f$ be a function mapping the set $X$ into the set $Y$. Let $A$ be an index set with elements $\alpha \in A$ and let two systems $\phi^{1}=\left\{\mathcal{F}_{\alpha}^{1}\right\}$ and $\phi^{2}=\left\{\mathcal{F}_{\alpha}^{2}\right\}$ of filters $\mathcal{F}_{\alpha}^{1}$ and $\mathcal{F}_{\alpha}^{2}$ be defined in $X$. Let a perfect topology $\mathfrak{I}$ be given on the set $A$. 
We say that the system $\phi^{1}$ has property $P$ with respect to $\phi^{2}$ and the topology $J$ if for every $F_{\alpha_{0}}^{1} \in \mathcal{F}_{\alpha_{0}}^{1}$ there is a neighborhood $N_{\alpha_{0}}$ of $\alpha_{0}$ such that every $F_{\alpha}^{2}$ with $\alpha \in N_{\alpha_{0}}$ and $\alpha \neq \alpha_{0}$ contains a set $F_{\alpha}^{2} \subseteq F_{\alpha_{0}}^{1}$. Similarly we speak of $\phi^{1}$ and $\phi^{2}$ having property $P$ with respect to the topologies $J_{12}$ and $J_{21}$. For instance if $X=A$ is the real line we can define for every $x=\alpha \in X=A$ the filter $\mathcal{F}_{x}^{-}$to be the filter generated by the family of open intervals $x-\delta<\xi \leqq x$ where $\delta>0$ is arbitrary and similarly we define $\mathcal{F}_{x}^{+}$to be the filter generated by the family of open intervals $x \leqq \xi<x+\delta$. Then the system $\phi^{+}$has property $P$ with respect to $\phi^{-}$and the topology $\mathrm{J}^{-+}$of the real line $X$ which is generated by the neighborhoods $N_{x}=[\xi / x \leqq \xi<x+\delta]$. Similar statement holds also when the ordering of $\phi^{-}$and $\phi^{+}$is reversed.

In the first theorem we choose $A$ to be a noncountable subset of the set $X$. The elements of $A$ will be denoted by $x$. We suppose that $x$ is contained in every set $F_{x}^{2} \in \mathcal{F}_{x}^{2}$ for every $x \in A$.

TheOREM 1. Suppose that the filter system $\phi^{1}$ has property $P$ with respect to $\phi^{2}$ and the perfect topology $\mathfrak{J}$, and suppose that a uniform structure $\mathcal{U}^{y}$ is given on $Y$ which satisfies the first axiom of countability. Then the set of those points $x \in A$ at which $f$ is continuous with respect to $\mathcal{F}_{x}^{1}$ and discontinuous with respect to $\mathcal{F}_{x}^{2}$ is countable.

There are a number of special cases and applications of this theorem which will be discussed in $\$ 7$. Here we mention only the examples when $X=Y$ is the real line, the filter systems are $\phi^{-}$and $\phi^{+}$with $A=X$, and the perfect topology $\mathfrak{I}$ is one of the half-open interval topologies $\mathrm{J}^{-+}$and $\mathrm{J}^{+-}$. Applying Theorem 1 with both choices of $I$ we see that the set of those points where $f$ is discontinuous but continuous either from the left or from the right is a countable subset of $X$.

Now we make further restrictions on the structures given on the sets $X$ and $Y$. We suppose that there is a uniform structure $\mathcal{u}^{x}$ defined on $X$ which satisfies the first axiom of countability. As earlier we suppose that two systems of filters $\phi^{1}=\left\{\digamma_{\alpha}^{1}\right\}$ and $\phi^{2}=\left\{\Im_{\alpha}^{2}\right\}$ are given on $X$ and a perfect topology is defined on the index set $A$. Here we consider only such filters $\mathcal{F}_{\alpha}^{i}$ which have the property that given any $U$ there are sets $F^{i}$ in $\mathcal{F}_{\alpha}^{i}$ satisfying $F^{i} \times F^{i} \subseteq U$ and given any $F_{\alpha}^{i} \in \mathcal{F}_{\alpha}^{i}$ there is a $U \in \mathcal{U}^{x}$ such that $F^{i} \subseteq F_{\alpha}^{i}$ for every $F^{i} \in \mathcal{F}_{\alpha}^{l}$ satisfying $F^{i} \times F^{i} \subseteq U$. Since $U^{x}$ satisfies the first axiom of countability these hypotheses are equivalent to the following: $F_{\alpha}^{t}$ has a countable base and it is a Cauchy filter.

We say that $\phi^{1}$ has property $\pi$ with respect to $\phi^{2}, \mathfrak{I}$ and $\mathcal{U}^{x}$ if given any $\alpha_{0} \in A$, a set $F_{\alpha_{0}}^{2} \in \mathcal{F}_{\alpha_{0}}^{2}$ and a vicinity $U \in \mathcal{U}^{x}$ there is a neighborhood $N_{\alpha_{0}}$ of $\alpha_{0}$ such that for every $\alpha \in N_{\alpha_{0}} \alpha \neq \alpha_{0}$ the set $F_{\alpha_{0}}^{2}$ intersects some $F_{\alpha}^{1}$ satisfying $F_{\alpha}^{1} \times F_{\alpha}^{1} \subseteq U$. We can also speak about $\phi^{1}$ and $\phi^{2}$ having property $\pi$ with respect to the topologies $J_{12}$ and $J_{21}$ and the uniform structure $u^{x}$. We shall prove the following: 
THEOREM 2. Suppose that the filter system $\phi^{1}$ has property $\pi$ with respect to $\phi^{2}, \Im$ and $\mathcal{u}^{x}$ and suppose that a separated uniform structure $\mathcal{u}^{y}$ is given on $Y$ such that the uniform space defined by $\mathfrak{u}^{y}$ is compact. Then the set of those indices $\alpha$ for which $f\left[\mathfrak{F}_{\alpha}^{1}\right] \cap f\left[\mathfrak{F}_{\alpha}^{2}\right]=0$ is countable.

As a simple illustration let us take Miss Schmeiser's theorem: As $X$ we take the Euclidean plane and as $A$ a fixed straight line in $X$. For every $a \in A$ we consider a half line $l_{\alpha}^{i}$ with end point $\alpha$ and forming a fixed angle $\gamma^{i}$ with $A$. The filter $\mathcal{F}_{\alpha}^{i}$ is defined by the set of all open segments of $l_{\alpha}^{i}$ one of whose end points is $\alpha$. If $\gamma^{1} \neq \gamma^{2}$ and $l_{\alpha}^{1}$ and $l_{\alpha}^{2}$ belong to the same fixed half plane determined by $A$ for every $\alpha \in A$ then the filter systems $\phi^{1}$ and $\phi^{2}$ have property $\pi$ with respect to the topology $\mathrm{J}^{-+}$of the line $A$ and the ordinary uniform structure of $X$. Hence by Theorem 2 the set of those points $\alpha \in A$ for which real valued function $f$ defined on $X$ does not have a common interlimit as $\xi \rightarrow \alpha$ along $l_{\alpha}^{1}$ and $l_{\alpha}^{2}$ is a countable set.

THEOREm 3. Suppose that there is a uniform structure given on the sets $X$ and $Y$ such that $\mathcal{U}^{x}$ and $\mathfrak{u}^{y}$ satisfy the first axiom of countability and the uniform topology induced on $X \times Y$ by $\mathcal{u}^{x}$ and $\mathfrak{u}^{y}$ is a perfect topology. Then every function $f$ mapping $X$ into $Y$ is intercontinuous nearly everywhere in $X$, i.e. $f(x) \in f\left[\odot_{x}\right]$ for all but a countable set of points of $X$.

If $X$ and $Y$ are finite dimensional Euclidean spaces then the conditions are clearly satisfied and we obtain Bonferroni's result. Finally we can prove an extension of Young's theorem:

ThEOREM 4. Suppose that the filter system $\phi^{1}$ has the following property: Given any $F_{\alpha_{0}}^{1} \in \mathcal{F}_{\alpha_{0}}^{1}$ there is a neighborhood $N_{\alpha_{0}} \in J$ such that for every $\alpha \in N_{\alpha_{0}}$; $\alpha \neq \alpha_{0}$ there is a $F_{\alpha}^{2} \in \mathcal{F}_{\alpha}^{2}$ satisfying $F_{\alpha}^{2} \subseteq F_{\alpha_{0}}^{1}$. Then the set of those points $\alpha \in A$ for which

is countable.

$$
\left(\mathcal{F}_{\alpha}^{1}\right) \lim \sup f(\xi) \neq\left(\mathcal{F}_{\alpha}^{2}\right) \lim \sup f(\xi)
$$

Several special cases and applications of these theorems will be discussed after the proofs.

4. The proof of Theorem 1. We give an indirect proof. Let us suppose that there exists a noncountable subset $A_{1}$ of $A$ such that at each point $x \in A_{1}$ $f$ is continuous with respect to the filter $\mathcal{F}_{x}^{1}$ and is discontinuous with respect to the filter $\mathcal{F}_{x}^{2}$.

Let $f(x)$ be abbreviated by $y$ and similarly let $f(\xi)$ be denoted by $\eta$ whenever this abbreviation does not cause confusion. Since, at every $x \in A_{1}, f$ is discontinuous with respect to $\mathscr{F}_{x}^{2}$ there exist for each $x \in A_{1}$ a neighborhood $N_{y}$ of $y=f(x)$ such that for every $F_{x}^{2} \in \mathcal{F}_{x}^{2}$ there is at least one $\xi \in F_{x}^{2}$ for which $f(\xi) \notin N_{y}$. The neighborhoods $N_{y}$ are derived from the uniform structure $\mathcal{u}^{y}$ and so we have proved that there is a noncountable set $A_{1}$ such that for each 
$x \in A_{1}$ there is a vicinity $V$ with the property that $(y, \eta) \notin V$ for at least one point $\xi$ of every $F_{x}^{2}$.

We may choose the vicinity $V$ from a countable set of fundamental vicinities $V_{n}$ which form a countable base for the uniform structure $\mathfrak{u}^{y}$. The set $A_{1}$ is not countable and the number of choices for $V=V_{n}$ is at most denumerably infinite. Hence we can select a suitable subset $A^{*}$ of $A_{1}$ and a fixed vicinity $V$ such that the following statements hold:

(i) $A^{*}$ is a noncountable subset of $A$.

(ii) $f$ is continuous with respect to $\mathcal{F}_{x}^{1}$ at every point $x \in A^{*}$.

(iii) There is a fixed vicinity $V \in \mathcal{U}^{y}$ with the property that for each $x \in A^{*}$ there is $a \xi$ in every $F_{x}^{2} \in \mathcal{F}_{x}^{2}$ such that $\xi \neq x$ and $(y, \eta) \notin V$.

By the hypothesis of the theorem there is a topology $\mathfrak{I}$ on the set $A$ such that every noncountable subset of $A$ has a point of accumulation which belongs to this subset. Hence by (i) the set $A^{*}$ has a point of accumulation $x_{0}$ with respect to the topology $\Im$ which belongs to $A^{*}$ itself. Therefore by (ii) the function $f$ is continuous at $x_{0}$ with respect to the filter $\mathcal{F}_{x_{0}}^{1}$.

The continuity of $f$ at $x_{0}$ with respect to $\mathcal{F}_{x_{0}}^{1}$ means that given any $N_{y_{0}}$ there is a $F_{x_{0}}^{1}$ such that $\eta=f(\xi)$ belongs to $N_{\nu_{0}}$ for every $\xi \in F_{x_{0}}^{1}$. Since the neighborhoods of the uniform topology on $Y$ are derived from the vicinities $W$ of the uniform structure $\mathcal{u}^{y}$, to every $N_{y_{0}}$ there corresponds a $W \in \mathcal{U}^{y}$ such that $\eta \in N_{y_{0}}$ if and only if $\left(y_{0}, \eta\right) \in W$. Consequently the continuity of $f$ at $x_{0}$ with respect to the filter $\mathcal{F}_{x_{0}}^{1}$ can be stated in terms of the vicinities: Given any $W \in \mathcal{U}^{y}$ there exists a $F_{x_{0}}^{1}$ such that for every $\xi \in F_{x_{0}}^{1}$ we have $\left(y_{0}, \eta\right) \in W$.

We shall consider one vicinity $W$ chosen in a suitable way: As earlier, let $V$ denote the fixed vicinity which occurs in (iii). For this particular $V \in \mathcal{U}^{y}$ we can find by Lemma 1 a symmetric vicinity $W$ satisfying $W \circ W \subseteq V$. Having fixed $W$ we determine the set $F_{x_{0}}^{1}$ such that $\left(y_{0}, \eta\right) \in W$ for every $\xi \in F_{x_{0}}^{1}$. Therefore we have

(a) The vicinity $W$ is symmetric, $W \circ W \subseteq V$ and $\left(y_{0}, \eta\right) \in W$ for every $\xi \in F_{x_{0}}^{1}$.

Now we apply the second part of the hypothesis of the theorem: We can choose a neighborhood $N_{x_{0}}$ of the point $x_{0}$ in the topology $J$ such that for every $x ; x \neq x_{0}, x \in N_{x_{0}}$ there is an $F_{x}^{2}$ contained in $F_{x_{0}}^{1}$. Since $x_{0}$ is a point of accumulation of $A^{*}$ we can find a point $x$ such that $x \in A^{*}$ and also $x \in N_{x_{0}}$. First we fix such a point $x$ and then determine the set $F_{x}^{2} \subseteq F_{x_{0}}^{1}$. By hypothesis $x \in F_{x}^{2}$. Therefore we have

(b) The point $x \in A^{*}$ is contained in $F_{x}^{2}$ and also in $F_{x_{0}}^{1}$.

Now it is easy to arrive at a contradiction: On the one hand $x \in A^{*}$ and so by (iii) there is a $\xi \in F_{x}^{2}$ such that $(y, \eta) \notin V$. By (a) we have $W \circ W \subseteq V$ and so $(y, \eta) \notin W \circ W$. On the other hand by (a) we have $\left(y_{0}, \eta\right) \in W$ and by (b) we may apply (a) with $\xi=x$ and obtain $\left(y_{0}, y\right) \in W$. By the symmetry of $W$ also $\left(y, y_{0}\right) \in W$ and so using $\left(y_{0}, \eta\right) \in W$ we obtain $(y, \eta) \in W \circ W$. This is a contradiction and so the theorem is proved.

5. Proof of Theorem 2. We are going to prove the theorem by contradic- 
tion. We assume that the intersection of the nonvoid sets $f\left[\mathfrak{F}_{\alpha}^{1}\right]$ and $f\left[\mathfrak{F}_{\alpha}^{2}\right]$ is empty for a noncountable infinite set $A_{1}$ of indices $\alpha \in A$. Using Lemma 2 we choose countable bases of $\mathcal{u}^{x}$ and $\mathcal{u}^{y}$, say $\left\{U_{n}\right\}$ and $\left\{V_{n}\right\}$ so that the vicinities $U_{n}$ and $V_{n}$ are symmetric and $U_{1} \supseteq U_{2} \supseteq \cdots$ and $V_{1} \supseteq V_{2} \supseteq \cdots$. We consider a finite subdivision $\mathfrak{N}_{n}=\mathfrak{N}_{V_{n}}$ of $Y$ with meshes $M_{n}^{k}=M_{V_{n}}^{k}$. The existence of such subdivisions is assured by the compactness and by Lemma 3. For each fixed $\alpha \in A_{1}$ let $E_{n \alpha}$ be the union of those meshes $M_{n}^{k}$ for which there is at least one point such that $f(\xi) \in M_{n}^{k}$ and $\xi \in F_{\alpha}^{1}$ for some $F_{\alpha}^{1}$ satisfying $F_{\alpha}^{1} \times F_{\alpha}^{1} \subseteq U_{n}$.

The set $A_{1}$ is not countable and so there exists a noncountable subset $A_{2}$ of $A_{1}$ and a fixed set $E_{n}$ for each $n=1,2, \cdots$ which is the union of meshes $M_{n}^{k}$ such that for every $\alpha \in A_{2}$ we have $E_{n \alpha}=E_{n}$. Let $\bar{E}_{n}$ denote the closure of the set $E_{n}$. We shall prove the following statement:

There is a noncountable subset $A^{*}$ of $A_{2}$ and a fixed index $n$ such that the intersection of $\bar{E}_{n}$ and $f\left[\mathfrak{F}_{\alpha}^{2}\right]$ is empty for every $\alpha \in A^{*}$.

Since there are denumerably many possibilities for $n$ it is sufficient to find an index $n$ for every $\alpha \in A_{2}$ such that $\bar{E}_{n} \cap f\left[\mathscr{F}_{\alpha}^{2}\right]$ is empty. On the contrary let us assume that for some $\alpha \in A_{2}$ and for every index $n$ there is a $y_{n} \in Y$ with the property that $y_{n} \in \bar{E}_{n}$ and $y_{n} \in f\left[\mathcal{F}_{\alpha}^{2}\right]$. Then there is a $y_{n}^{\prime} \in E_{n}$ such that $\left(y_{n}, y_{n}^{\prime}\right) \in V_{n}$. Of course $y_{n}^{\prime} \in M_{n}^{k}$ for some index $k$. According to the definition of $E_{n}$ there is a $\xi_{n}$ such that $\eta_{n}=f\left(\xi_{n}\right) \in M_{n}^{k}$ and $\xi_{n} \in F_{n \alpha}^{1}$ for some $F_{n \alpha}^{1}$ with $F_{n \alpha}^{1} \times F_{n x}^{1} \subseteq U_{n}$. Since $M_{n}^{k} \times M_{n}^{k} \subseteq V_{n}$ by the definition of the subdivision $\mathscr{N}_{n}$, we see that $\left(y_{n}^{\prime}, \eta_{n}\right) \in V_{n}$ and so $\left(y_{n}, \eta_{n}\right) \in V_{n} \circ V_{n}$.

By hypothesis $f\left[\mathcal{F}_{\alpha}^{2}\right]$ is compact and so the set of points $y_{n}$ has a point of accumulation $y \in f\left[\mathfrak{F}_{\alpha}^{2}\right]$. Thus for every $m$ there is at least one index $n=n_{m}>m$ such that $\left(y, y_{n}\right) \in V_{m}$. Since also $\left(y_{n}, \eta_{n}\right) \in V_{n} \circ V_{n}$ we have $\left(y, \eta_{n}\right)$ $\in V_{m} \circ V_{m} \circ V_{m}$, where $\eta_{n}=f\left(\xi_{n}\right)$ and $\xi_{n} \in F_{n \alpha}^{1}$ with $F_{n \alpha}^{1} \times F_{n \alpha}^{1} \subseteq U_{n}$.

It follows that $y$ is a point of the set $f\left[\mathcal{F}_{\alpha}^{1}\right]$ : In fact by Lemma 1 for every $V$ given in advance there is a $V_{m}$ such that $V_{m} \circ V_{m} \circ V_{m} \subseteq V$. Moreover given any $F \in \mathcal{F}_{\alpha}^{1}$ there is a $U \in \mathcal{U}^{x}$ such that $F_{\alpha}^{1} \subseteq F$ for every $F_{\alpha}^{1}$ satisfying $F_{\alpha}^{1} \times F_{\alpha}^{1} \subseteq U$. Hence for a sufficiently high index $n$ we have $F_{n \alpha}^{1} \subseteq F$, so that $\xi_{n} \in F$ and $\left(y, \eta_{n}\right) \in V$. Therefore $y \in f\left[\mathfrak{F}_{\alpha}^{1}\right]$. This is a contradiction because on the one hand we also have $y \in f\left[\mathcal{F}_{\alpha}^{2}\right]$ and on the other hand $f\left[\mathcal{F}_{\alpha}^{1}\right] \cap f\left[\mathcal{F}_{\alpha}^{2}\right]$ is empty for every $\alpha \in A_{2}$. This proves the statement.

Now we can arrive at a contradiction: Since the topology $J$ is perfect and $A^{*}$ is not countable there is an index $\alpha_{0} \in A^{*}$ which is a point of accumulation of $A^{*}$ with respect to the topology $\Im$. Given any $U_{m}$ of the decreasing sequence $\left\{U_{m}\right\}$ of symmetric fundamental vicinities we choose $F_{m \alpha_{0}}^{2} \in \mathcal{F}_{\alpha_{0}}^{2}$ such that $F_{m \alpha_{0}}^{2} \times F_{m \alpha_{0}}^{2} \subseteq U_{m}$. Given $F_{m \alpha_{0}}^{2}$ by hypothesis there is a neighborhood $N_{\alpha_{0}}$ of $\alpha_{0}$ such that the following statement holds for the fixed vicinity $U_{n}$ which corresponds to the fixed set $\bar{E}_{n}$. For every $\alpha \in N_{\alpha_{0}} ; \alpha \neq \alpha_{0}$ there is an $F_{\alpha}^{1}$ with the properties $F_{\alpha}^{1} \times F_{\alpha}^{1} \subseteq U_{n}$ and $F_{\alpha}^{1} \cap F_{m \alpha_{0}}^{2} \neq 0$. Since $\alpha_{0}$ is a point of accumulation of $A^{*}$ there is an $\alpha \in N_{\alpha_{0}}$ such that $\alpha \in A^{*}$. Let $\xi_{m}$ be a common point of $F_{\alpha}^{1}$ and $F_{m \alpha_{0}}^{2}$. Then $\eta_{m}=f\left(\xi_{m}\right) \in \bar{E}_{n}$. 
The compactness of the closed set $\bar{E}_{n}$ implies that the sequence $\left\{\eta_{m}\right\}$ has a point of accumulation $y$ which belongs to $\bar{E}_{n}$. We can easily show that $y$ also belongs to $f\left[\mathfrak{F}_{\alpha_{0}}^{2}\right]$. This leads to a contradiction because $\alpha_{0} \in A^{*}$ and so $\bar{E}_{n} \cap f\left[\mathcal{F}_{\alpha_{0}}^{2}\right]$ is empty. In fact given any $F \in \mathcal{F}_{\alpha_{0}}^{2}$ there is a $U \in \mathcal{U}^{x}$ such that $F_{\alpha_{0}}^{2} \subseteq F$ for every $F_{\alpha_{0}}^{2}$ satisfying $F_{\alpha_{0}}^{2} \times F_{\alpha_{0}}^{2} \subseteq U$. Therefore if we choose $m$ such that $U_{m} \subseteq U$ it follows by $F_{m \alpha_{0}}^{2} \times F_{m \alpha_{0}}^{2} \subseteq U_{m}$ that $F_{m \alpha_{0}}^{2} \subseteq F . \xi_{m} \in F_{m \alpha_{0}}^{2}$ and so $\xi_{m} \in F$ for every $m$ whenever $U_{m} \subseteq U$. Let also $V \in \mathcal{U}^{\nu}$ be given. Since $y$ is a point of accumulation of $\left\{\eta_{m}\right\}$ we have $\left(y, \eta_{m}\right) \in V$ for an infinity of indices $m$. We proved that $\xi_{m} \in F$ with the possible exception of at most finitely many indices $m$. Hence there are infinitely many $m$ 's for which both $\xi_{m} \in F$ and $\left(y, \eta_{m}\right) \in V$ holds. Consequently $y$ belongs to $f\left[\mathfrak{F}_{\alpha_{0}}^{2}\right]$ and so the theorem is proved.

6. Proofs of Theorems 3 and 4 . We prove the results by contradiction. First let us suppose that there is a noncountable set $X_{1} \subseteq X$ such that $y$ $=f(x) \notin f\left[\rho_{x}\right]$ for every $x \in X_{1}$. Then there exist $U \in \mathcal{U}^{x}$ and $V \in \mathcal{U}^{y}$ for every $x \in X_{1}$ with the property that $(y, \eta) \notin V$ for every $\xi$ satisfying $(x, \xi) \notin U$. By Lemma 2 there are decreasing sequences $\left\{U_{m}\right\}$ and $\left\{V_{m}\right\}$ of symmetric fundamental vicinities of $\mathcal{U}^{x}$ and $\boldsymbol{u}^{\nu}$. Hence we may suppose that $U$ and $V$ are chosen from the elements of the sequences $\left\{U_{m}\right\}$ and $\left\{V_{m}\right\}$ respectively. Since on the one hand the possibilities for such a $U, V$ pair form a denumerable set and on the other hand $X_{1}$ is not countable there are fixed vicinities $U_{n}$ and $V_{p}$ and a noncountable subset $X_{2}$ of $X_{1}$ such that $(y, \eta) \notin V_{p}$ for every $\xi$ satisfying $(x, \xi) \in U_{n}$.

Now we consider the set $S$ of ordered pairs $(x, y) \in X \times Y$ where $x \in X_{2}$ and $y=f(x)$. This set is not countable and so by the hypothesis of the theorem there is a point of accumulation $\left(x_{0}, y_{0}\right)$ which belongs to $S$. Hence choosing the neighborhood of $\left(x_{0}, y_{0}\right)$ which is determined by the vicinities $U_{n}$ and $V_{p}$ we see that there is a pair $(x, y) \in S$ with the properties $\left(x, x_{0}\right) \in U_{n},\left(y, y_{0}\right) \in V_{p}$. This is a contradiction because on the one hand by $(x, y) \in S$ we have $x \in X_{2}$ and so $\left(y, f\left(x_{0}\right)\right) \notin V_{p}$ but on the other hand by $\left(x_{0}, y_{0}\right) \in S$ we have $y_{0}=f\left(x_{0}\right)$. This completes the proof of Theorem 3.

Proof of Theorem 4. For the sake of simplicity we shall use the notation $\Lambda_{\alpha}^{i}=\left(\mathcal{F}_{\alpha}^{1}\right)-\lim \sup f(\xi)$. Let us suppose that $\Lambda_{\alpha}^{1} \neq \Lambda_{\alpha}^{2}$ for a noncountable subset $A_{1}$ of indices $\alpha \in A$. By a suitable choice of the superscripts $i=1,2$ we can make $\Lambda_{\alpha}^{1}<\Lambda_{\alpha}^{2}$ for a noncountable subset $A_{2}$ of $A_{1}$. Since the set $A_{2}$ is not countable there is an $\epsilon>0$ and a noncountable subset $A_{3}$ of $A_{2}$ such that $\Lambda_{\alpha}^{1}+\epsilon<\Lambda_{\alpha}^{2}$ for every $\alpha \in A_{3}$. We consider the set of values $\Lambda_{\alpha}^{2}$ counting each value with its multiplicity in $\alpha$. This set is not countable and so there is a real number $\Lambda^{2}$ and a noncountable subset $A^{*}$ of $A_{3}$ with the property that $\left|\Lambda^{2}-\Lambda_{\alpha}^{2}\right|<\epsilon / 3$ for every $\alpha \in A^{*}$.

The topology $J$ is perfect and so there is a point of accumulation $\alpha_{0}$ of the set $A^{*}$ which belongs to $A^{*}$. By the hypothesis of the theorem, given $F_{\alpha_{0}}^{1} \in \mathcal{F}_{\alpha_{0}}^{1}$ there is a neighborhood $N_{\alpha_{0}}$ such that for every $\alpha \in N_{\alpha_{0}} ; \alpha \neq \alpha_{0}$ we 
have $F_{\alpha}^{2} \subseteq F_{\alpha_{0}}^{1}$ for some $F_{\alpha}^{2} \in \mathcal{F}_{\alpha}^{2}$. By the definition of $\Lambda_{\alpha}^{2}$ there is a $\xi \in F_{\alpha}^{2}$ such that $f(\xi)>\Lambda_{\alpha}^{2}-\epsilon / 3$. Therefore $f(\xi)>\Lambda^{2}-2 \epsilon / 3$ with $\xi \in F_{\alpha_{0}}^{1}$ so $\left(F_{\alpha_{0}}\right)$ -lub $f(\xi)>\Lambda^{2}-2 \epsilon / 3$. The set $F_{\alpha_{0}}^{1} \in \mathcal{F}_{\alpha_{0}}^{1}$ being arbitrary we see that $\Lambda_{\alpha_{0}}^{1}$ $\geqq \Lambda^{2}-2 \epsilon / 3$. Since $\alpha_{0} \in A^{*}$ we have $\Lambda_{\alpha_{0}}^{1}>\Lambda_{\alpha_{0}}^{2}-\epsilon$, which is in contradiction to $\Lambda_{\alpha_{0}}^{1}+\epsilon<\Lambda_{\alpha_{0}}^{2}$.

7. Special cases and applications of Theorem 1. First we modify Theorem 1 to obtain a more symmetric form: Suppose that a countable set $\left\{\phi^{i}\right\}$ of filter systems $\phi^{i}$ is given in $X$ in such a way that to every point $x \in A$ there corresponds a filter $\mathcal{F}_{x}^{i} \in \phi^{i}$ where the index set $A$ is a noncountable subset of $X$. Let a perfect topology $\Im_{i j}$ be defined on $A$ for every ordered pair of indices $i, j=1,2, \cdots$. Suppose that $\phi^{i}$ and $\phi^{i}$ have property $P$ with respect to the topologies $J_{i j}$ and $J_{j i}$ for every $i, j=1,2, \cdots$ and $x \in F_{x}^{i}$ for every $F_{x}^{i} \in \mathcal{F}_{x}^{i}$ and every $i$ and $x \in A$. Using Theorem 1 we obtain the following proposition:

If for every $x \in A$ there is an index $i$ such that $f$ is continuous at $x$ with respect to $\mathfrak{F}_{x}^{i}$ then for nearly every $x \in A$ fis continuous with respect to every filter $\mathfrak{F}_{x}^{i}(i=1,2, \cdots)$.

The conclusion can be stated in a different form by using the concept of the greatest lower bound of a family of filters. For every $x \in A$ we define the greatest lower bound of the family $\left\{\mathcal{F}_{x}^{i}\right\}$ to be the set $\mathcal{F}_{x}=\operatorname{glb} \mathcal{F}_{x}^{i}$ of those sets $F_{x} \subseteq X$ which include at least one set $F_{x}^{i}$ from every filter $\mathcal{F}_{x}^{i}$. It is easy to see that $\mathcal{F}_{x}$ satisfies the axioms (1), (2) and (3) of a filter. By the foregoing proposition given any $x \in A$ which is not an exceptional point and given any $N_{y} \subseteq Y$ there is an $F_{x}^{i}$ for every $i=1,2, \cdots$ such that $\eta \in N_{y}$ for all $\xi \in F_{x}^{i}$. Let $F_{x}=\bigcup_{i} F_{x}^{i}$. Clearly $\eta \in N_{y}$ for every $\xi \in F_{x}$ and $F_{x} \in \mathcal{F}_{x}$. Then under the same hypothesis as in the preceding proposition the conclusion now reads: $f$ is continuous with respect to $\mathcal{F}_{x}=$ glb $\mathfrak{F}_{x}^{i}$ nearly everywhere in $A$.

Now we make further restrictions on the systems $\phi^{i}$ : We start from a countable family $\left\{J^{i}\right\}$ of topologies $J^{i}$ defined on the set $X$. By definition the greatest lower bound of the family $\left\{J^{i}\right\}$ is the topology $J=$ glb $J^{i}$ whose open sets $O \in J$ are open in every $J^{i}$. We define the filter system $\phi^{i}=\left\{\mathcal{P}_{x}^{i}\right\}$ to be the set of all filters $\mathcal{P}_{x}^{i}$ generated by the neighborhoods $N_{x}^{i} \in \mathfrak{J}^{i}$ of $x \in A$ so that $F_{x}^{i} \in \mathcal{\rho}_{x}^{i}$ if and only if $N_{x}^{i} \subseteq F_{x}^{i}$ for some open set $N_{x}^{i} \in J^{i}$.

If $O_{x}$ is an open set in $J$ which contains $x$ then $O_{x} \in J^{i}$ and so $O_{x} \in \mathcal{P}_{x}^{i}$ for every $i$. Therefore $O_{x}$ is a set of the filter $\mathcal{F}_{x}=\operatorname{glb} \mathscr{P}_{x}^{i}$. This implies that in general $\mathcal{P}_{x} \subseteq \mathcal{F}_{x}$ where $\mathcal{P}_{x}$ denotes the filter generated by the neighborhoods $N_{x}$ of $x$ in $J$. For every $i, j$ let the systems $\phi^{i}$ and $\phi^{i}$ have property $P$ with respect to some suitable perfect topologies $\Im_{i j}$ and $\Im_{j i}$ defined on $A$, and at every $x \in A$ let $f$ be continuous with respect to some topology $J^{i}$ where depends on $x$. Then by the above corollary of Theorem 1 the function $f$ is continuous with respect to the filter system $\phi=\left\{\mathscr{F}_{x}\right\}$ at nearly all $x \in A$. This last conclusion however does not imply the continuity of $f$ with respect to the topology $\mathfrak{J}$ because only $\mathcal{P}_{x} \subseteq \mathcal{F}_{x}$ is known.

There are a number of important instances when the filters $\mathcal{P}_{x}$ and $\mathcal{F}_{x}$ 
coincide. In view of $\mathcal{P}_{x} \subseteq \mathcal{F}_{x}$ it is sufficient to show that $\mathcal{F}_{x} \subseteq \mathcal{P}_{x}$, i.e. every set $F_{x} \in \mathcal{F}_{x}$ contains some $V_{x} \in \mathcal{P}_{x}$. The simplest example of such topologies is furnished by the topologies $\mathrm{J}^{-+}$and $\mathrm{J}^{+-}$induced on the real line $X$ by the half open intervals $a \leqq \xi<b$ and $a<\xi \leqq b$ respectively. Here $\mathfrak{J}=$ glb $\left(3^{-+}, \mathrm{J}^{+-}\right)$ is the usual topology of the real line and the conclusion gives the second corollary of Young's theorem which was discussed in the introduction. For the topologies $\mathrm{J}^{+-}$and $\mathrm{J}^{-+}$are perfect and can serve as the topologies $\mathfrak{J}^{i j}$ and $\mathfrak{J}^{j i}$ of the hypothesis.

Similar situations arise in the plane. First we introduce a notation: We consider the circle with center $x \in X$ and radius $\rho$. Given a direction $\boldsymbol{d}$ in $X$ and any angle $\alpha(0<\alpha<2 \pi)$ we let $S_{x}(\rho)=S_{x}(d, \alpha, \rho)$ denote the sector with central angle $\alpha$ pointing in the direction $d$. We agree that the vertex $x$ belongs to $S_{x}(\rho)$ and no other boundary point of the sector belongs to the plane set $S_{x}(\rho)$. We say that a topology $J$ in the plane is a $(d, \alpha)$-topology if every neighborhood $N_{x} \in J$ contains some $S_{x}(\boldsymbol{d}, \alpha, \rho)$. For instance the family of all sets $S_{x}(\boldsymbol{d}, \alpha, \rho)$ for fixed $(\boldsymbol{d}, \alpha)$ and arbitrary $x \in X, \rho>0$ forms a base of a $(d, \alpha)$-topology; it is the finest among all $(d, \alpha)$-topologies. Obviously a $(d, \alpha)$-topology is necessarily a $(d, \beta)$-topology for every $\beta<\alpha$. We prove the following lemma:

Every $(\boldsymbol{d}, \alpha)$-topology with $\alpha>\pi$ is perfect. For every $\beta \leqq \pi$ there is $a(\boldsymbol{d}, \beta)$ topology which is not perfect.

Proof. We give an indirect proof. Suppose that there is a noncountable set $A \subseteq X$ such that no point $x \in A$ is a point of accumulation of $A$. Then we can associate with every $x \in A$ a sector $S_{x}\left(\rho_{x}\right)$ whose radius may depend on $x$ such that $S_{x}\left(\rho_{x}\right)$ contains no points of $A$ except $x$. The set $A$ is not countable and so there is a noncountable subset $A^{*}$ of $A$ and a common radius $\rho>0$ with the property that the only common point of $S_{x}(\rho)$ and $A^{*}$ is $x$. Let $x_{1}, x_{2} \in A^{*}$ and let the indices be chosen such that $x_{2}$ is not farther in the direction $d$ than $x_{2}$. Then $x_{1} \in S_{x}(\boldsymbol{d}, \alpha, \rho)$ and $\alpha>\pi$ imply that $x_{2}$ is outside the circle about $x_{1}$ with radius $\rho$. Consequently the distance between any two points $x_{1} \neq x_{2}$ of $A^{*}$ is at least $\rho$. Since the usual topology of the plane is perfect we have a contradiction.

The second statement of the lemma can be proved by considering the $(\boldsymbol{d}, \beta)$-topology whose base is $S_{x}(\boldsymbol{d}, \beta, \rho)$ where $\rho>0$ and $x \in X$ are variables. Let $A$ be any straight line which is perpendicular to $d$. Give any $x \in A$ consider the neighborhood $S_{x}(d, \beta, \rho)$ of $x$. Since $\beta \leqq \pi, S_{x}(d, \beta, \rho)$ contains no other point of $A$ besides the vertex $x$. Hence $x$ is not a point of accumulation of $A$.

Now we consider a denumerable family of unit vectors $\boldsymbol{d}^{i}$ which is dense in the space of all unit vectors, i.e. given $\boldsymbol{d}$ and $\epsilon>0$ there is an index $i$ such that $\boldsymbol{d} \cdot \boldsymbol{d}^{i}>1-\epsilon$. Let $J^{i}(i=1,2, \cdots)$ be a $\left(\boldsymbol{d}, \alpha^{i}\right)$-topology with $\alpha^{i}>\pi$. Then for every $i, j=1,2, \cdots$ the filter system $\phi^{i}=\left\{\mathcal{P}_{x}^{i}\right\}$ has property $P$ with respect to $\phi^{j}$ and the perfect topology $J^{i}$. Hence the hypotheses of our last prop- 
osition are satisfied and so if a function $f$ is continuous at every $x \in A$ with respect to at least one of the topologies $J^{i}$ then $f$ is continuous at nearly all points $x \in A$ with respect to $\mathcal{F}_{x}=$ glb $P_{x}^{i}$. Clearly every $F_{x} \in \mathcal{F}_{x}$ contains a circle with center $x$ and so $f$ is continuous in the usual sense nearly everywhere in $A$. We obtain the following result:

Let $f$ be a function mapping the plane into a uniform space $Y$ whose uniform structure $\mathcal{u}^{y}$ satisfies the first axiom of countability. If at every point $x$ of a noncountable subset $A$ of $X f$ is continuous with respect to some $(d, \alpha)$-topology where $\alpha>\pi$, then $f$ is continuous in the usual sense at nearly all $x \in A$.

The function $f$ is continuous at $x$ in the $(d, \alpha)$-topology if and only if the restriction of $f$ to the plane sector pointing in the direction $d$ and having central angle $\alpha$ is continuous at the vertex $x$. The result can be readily extended to finite dimensional Euclidean spaces.

8. Remarks on Theorem 2 and examples. It is obvious that the reasoning given in the beginning of $\$ 7$ concerning Theorem 1 can be repeated also in the present case. Hence we can consider a countable set $\left\{\phi^{i}\right\}$ of filter systems $\phi^{i}=\left\{F_{\alpha}^{i}\right\}$ where the index set $A=\{\alpha\}$ is not countable. We suppose that $\phi^{i}$ and $\phi^{i}$ have property $\pi$ with respect to some perfect topologies $J_{i j}$ and $\mathfrak{J}_{j i}$ defined on $A$. Then under the same hypotheses on $X$ and $Y$ as in Theorem 2 we have:

For nearly all choices of $\alpha \in A$ any two sets $f\left[\mathfrak{F}_{\alpha}^{\prime}\right]$ and $f\left[\mathfrak{F}_{\alpha}^{j}\right]$ have a nonzero intersection, i.e. $f$ has a common limiting value with respect to any two filters $\mathcal{F}_{\alpha}^{i}$ and $\mathfrak{F}_{\alpha}^{\jmath}$.

It is remarkable that in certain instances the countability condition on the index set $\{i\}$ can be relaxed. This is the situation, for example, in the problem treated by Miss Schmeiser. Let us consider all directions $d^{i}$ on the same side of the straight line $A$ in the plane $X$. Let us denote by $\mathcal{F}_{x}^{l}$ the filter generated by the segments which have $x$ as one endpoint and lie on the half line from $x$ in the direction $d^{i}$. If we suppose that there is a noncountable subset $A_{1}$ of $A$ such that for every $x \in A_{1}$ there exist two directions $d^{i}$ and $d^{j}$ with the property that the intersection of $f\left[\mathcal{F}_{\alpha}^{i}\right]$ and $f\left[\mathfrak{F}_{\alpha}^{j}\right]$ is empty, then we can find a noncountable subset $A_{2}$ of $A_{1}$ and an angle $\delta(0<\delta<\pi)$ such that for every $\alpha \in A_{2}$ the angle between the exceptional directions $d^{i} ; i=i(\alpha)$ and $\boldsymbol{d}^{j} ; j=j(\alpha)$ is greater than $\delta$.

The filter systems $\phi^{1}=\left\{F_{\alpha}^{i}\right\}$ and $\phi^{2}=\left\{\mathscr{F}_{\alpha}^{j}\right\}$ where $i=i(\alpha)$ and $j=j(\alpha)$ have property $\pi$ with respect to the topology $3^{-+}$and $3^{+-}$of the straight line $A$ and so the hypotheses of Theorem 2 are satisfied. On the other hand $f\left[\mathcal{F}_{\alpha}^{i}\right] \cap f\left[\mathfrak{F}_{\alpha}^{j}\right]=0$ for every $\alpha \in A_{2}$, i.e. the conclusion of Theorem 2 does not hold. Therefore there cannot be such a noncountable subset $A_{1}$ and we have:

For nearly all choices of $\alpha \in A$ the sets $f\left[\mathfrak{F}_{\alpha}^{i}\right]$ and $f\left[\mathfrak{F}_{\beta}^{j}\right]$ intersect for all directions $\boldsymbol{d}^{i}$ and $\boldsymbol{d}^{j}$ on the same side of $A$.

This result is Jarnik's improvement of the theorems of Blumberg and Miss Schmeiser. His proof is a direct proof but it makes extensive use of the 
special properties of the Euclidean plane. It is easy to repeat the foregoing reasoning to obtain similar improvements of Miss Schmeiser's result on function defined in Euclidean 3-space. Various applications of these results can be found in Blumberg's paper.

According to a later result of W. H. Young [17] on real valued functions defined on the real line the sets $f\left[\mathfrak{F}_{x}^{-}\right]$and $f\left[\mathfrak{F}_{x}^{+}\right]$coincide for nearly all $x \in X$. This theorem can also be extended to abstract spaces and it is again the property $P$ which matters. However we do not wish to discuss the problem in details.

It would be desirable to give an alternative characterization of perfect topological spaces which is equivalent in the same sense as the compactness in the Fréchet sense (using filters) and in the Alexandroff-Urysohn sense are equivalent. For uniform spaces several such characterizations can be obtained by using Zorn's lemma. This and related problems will be discussed in a future paper.

\section{BIBLIOGRAPHY}

1. W. H. Young, On the distinction of right and left at points of discontinuity, Quarterly Journal of Mathematics vol. 39 (1908) pp. 67-83.

2. G. Aumann, Reelle Funktionen, Berlin, Springer-Verlag, 1954, p. 236.

3. N. Bourbaki, Fonctions d'une variable réelle, Chapitre I, Paris, Hermann et Cie, 1949, p. 59.

4 C. Bonferroni, Una proprietà generale delle funsioni, Boll. Un. Mat. Ital. (3) vol. 8 (1953) pp. 384-390.

5. S. Saks, Theory of the integral, New York, Hafner, 1937, p. 261.

6. H. Blumberg, $A$ theorem on arbitrary functions of two variables with applications, Fund. Math. vol. 16 (1930) pp. 17-24.

7. M. Schmeiser, Some properties of arbitrary functions, Fund. Math. vol. 22 (1934) pp. 70-76.

8. V. Jarnik, Sur les fonctions de deux variables réelles, Fund. Math. vol. 27 (1936) pp. 147152.

9. E. J. McShane, Partial orderings and Moore-Smith limits, Amer. Math. Monthly vol. 59 (1952) pp. 1-11.

10. - A theory of convergence, Canadian Journal of Mathematics vol. 6 (1954) pp. 161-168.

11. R. G. Bartle, Nets and filters in topology, Amer. Math. Monthly vol. 62 (1955) pp. $551-$ 557.

12. F. Schmidt, Beitrage zur Filtertheorie II, Mathematische Nachrichten vol. 10 (1953) pp. 197-232.

13. J. L. Kelley, General topology, New York, Van Nostrand, 1955.

14. N. Bourbaki, Topologie générale, Chapitre II, Paris, Hermann et Cie., 1940.

15. G. Nöbeling, Grundlagen der analytischen Topologie, Berlin, Springer-Verlag, 1954.

16. C. Kuratowski, Topologie I, Monografie Matematyczne (Warszawa) 1948, pp. 133-134.

17. IV. H. Young, La symétrie de structure des fonctions de variables réelles, Bull. Sci. Math. 2 d ser. vol. 52 (1928) pp. 265-280.

Corvell I'Niversity

ITHACA, N. Y. 